\title{
Body mass index (BMI) and cognitive functions in later life
}

\begin{abstract}
Background: The findings from previous studies exploring the association between BMI and cognitive function in the elderly are conflicting. The purpose of the present study is twofold; to verify the association between BMI and cognitive functions and examine whether this association is impacted by height, when adjusted for possible covariates.

Methods: The data for this study, consisted of 2287 older adults aged 60 years and above, drawn from a nationally representative population-based survey entitled "Determinants of Wellness among Older Malaysians: A Health Promotion Perspective" conducted in 2009.

Results: The mean age of the respondents was $68.7(\mathrm{SD}=6.6)$ years. The average score of cognitive function, measured by MMSE was 24.5 ( $\mathrm{SD}=5.6$ ). About $40 \%$ of the respondents were classified as overweight. Results of the multiple linear regression analysis revealed a significant association between BMI and cognitive function (Beta=.10, p<.001). The Factorial ANCOVA revealed significant interaction effect between BMI and height on cognitive function $(\mathrm{F}=10.8, \mathrm{p}<.001)$, after adjusting for possible covariates.

Conclusion: The findings from the current study indicating the positive association between $\mathrm{BMI}$ and cognitive function depends on height, therefore it is suggested that short people might be targeted for dementia prevention.
\end{abstract}

Keyword: Aged; BMI; Cognitive function; Epidemiology; Mental health; Older adults 